\title{
Measurement of Trauma Based on Exponential Injury Severity Score from Anger Management
}

\section{Zharama M. Llarena}

PhD student, Gokongwei College of Engineering, De La Salle University Manila

\section{Abstract}

Anger is an emotional trauma experienced by a person inflicted with external harm, such as abusive words and experienced injustice by an individual. Its management can exhibit the degree of trauma felt by a person. This paper aimed to measure the symptoms of aggressiveness brought by a series of recall by the individual. Cognitivebehavioral intervention ( $\mathrm{CBI}$ ) was a tool used to mitigate post-traumatic symptoms brought by exposure risk to emotional harm. A 33-year-old Asian man had experienced moral injury to the extent of losing significant weight in a span of 8 months resulting from

Corresponding Author: Zharama M. Llarena zharllarena21@yahoo.com.ph

Received: 18 January 2019 Accepted: 24 March 2019 Published: 31 March 2019 Publishing services provided by Knowledge E

(c) Zharama M. Llarena. This article is distributed under the terms of the Creative Commons Attribution License, which permits unrestricted use and redistribution provided that the original author and source are credited.

Selection and Peer-review under the responsibility of the First ELEHIC Conference Committee. being obese to a normal body mass index (BMI) as a result of his anger management. Exponential Injury Severity Score (EISS) in reference to Abbreviated Injury Scale (AIS) system showed that the 33-year-old Asian man had suffered severe injury with a score of 5.29 near to a 6.0 maximal score. Thus, anger management using CBI can be utilized a person to release negative emotions resulting to optimum quality of life and health.

Keywords: trauma, anger management, emotional harm

\section{Introduction}

Individual hermeneutics that are comprised by actual, serious or threatened injury or death can directly lead to trauma. Traumatic episodes include persistent consequences of several impairments, such as occupational, psychological, life quality and functional disabilities. Physical trauma may possibly occur or may not occur simultaneously with psychological trauma, or it may independently happen. Similar to physical trauma, near death episodes of psychological trauma like massive fire can result to several impairments in psychological outcomes, namely, major depression, anxiety disorders, posttraumatic stress disorder, comparable to occupational, psychological, life quality and functional disabilities [1]. 
Psychological symptoms can occur simultaneously with somatic symptoms like depression, anger, anxiety, initial shock, inability to concentrate, a dazed sensation, loss of libido, lassitude, insomnia, modified appetite and weight and, in other events, sensations of despair, horror, helplessness, and recalling experiences. Even though there is an acknowledgement in progressive link between pain symptomatology and subject's mood, pain symptoms can be sustained or aggravated by persistent augmentation in anxiety, depression, or arousal level of mood related to psychological symptomatology. As an example, medico-legal evaluations running in one series of 50 consecutive assessments where pain was observed as a major symptom, exaggeration of disability was found in $98 \%$ of patients [2].

One of the fundamental emotions felt by majority of individuals in their daily lives is anger. It is a particular provocation responsive to an intense distress feeling. There are several means to show that people are angry such as when they are treated unfairly, unjustified by blamed action, kept from getting their goals or observed violation of cultural norms and when people tried to attack their innocent behavior. Anger, hostility and aggression are terms often utilized interchangeability. Although, anger, hostility and aggression can illustrate the cognitive, affective and behavioral components of the similar multidimensional construct as recognized by some researchers. Hence, three basic dimensions can be perceived using the construct such as first, affective, build-up of emotions like anger or loathing, second, cognitive, bombarded of negative thoughts regarding human nature, bitterness, and cynical doubt, and lastly, behavioral, described by several aggressive forms like physical or verbal hostility. All of these determinants are observed to be associated with each other with difference in intensity, frequency, and duration [3].

On a continuum existence in which the frequency, intensity and duration of hermeneutics along with subjective, physiological, interpretive, and behavioral expressive attributes, anger is a negative phenomenological experience resulting to remarkable emotional trauma. There are suggestions based on several studies that anger-related emotional trauma is usually exhibited in various physical and psychological issues. For instance, Williams et al. (2000) discovered that anger puts people in middle adulthood at risk for coronary heart disease and death. Several arguments have been raised that anger has the potential to mediate domicile violence and substance abuse disorders. Anger has negative effects that are not restricted to the aggressive person, although, behavior exhibiting aggressiveness has the potential to inflict harm or injury to other individuals [3]. 
Anger, as defined by Feindler (2006), has a various intensity ranges, starting from hurt feelings to rage, and duration, beginning from painful experiences to chronic hard sensations where negative perception of emotional state is felt by an individual. This negative experience makes the people to be more sensitive for aggressive reaction which is the major result of some past hurtful events and is commonly observed with unfriendly decision and subjective presumption. Behavioral reactions to anger comprise an immense range of responses in order to avoid anger source resulting to behavioral aggression [4]. Anger has been defined as a negative destructive emotion often related to sorrow, trouble, rage, and wrathe; as a subjective emotional state that involves the interrelationship of psychological components and cognitive appraisal; and a negative feeling state associated with cognitive distortion, physiologic changes, and behavioral reactions [5].

Anger expression may take various forms, constituting self-harm, violence, and usually, verbal and physical aggression. Anger accounts for the aggression production. Hence, anger results to do more danger than good, thus, it should be controlled [5].

The increasing trend in violence among adolescents had been of great concern. Violence is a form of physical aggression and it is usually an expression of anger. Aggression can be categorised into verbal and physical. Although anger is a common and natural emotion, or internal event, problems associated with inappropriate expression of anger remain among the most serious concerns of parents, educators, and the mental health community. Anger leads to aggression. When anger failed to be controlled it would lead to aggression. Anger and its expression represent a major public health problem for school-age and adolescents. Anger is associated with serious harm and in the worst case scenario, homicides may result. Further if an adolescent's anger occurs with aggression, a host of additional negative consequences may ensue. Anger and aggression, which are generally considered as negative, are disruptive and detrimental emotional and behavioral expressions in social encounters [5].

Anger management is a vital topic in violent crime prevention. Aggressive behaviors, either continuous or dangerous, require fast regulation and assessment. Recent studies associated with other investigations facilitated by various participants, scientists, and determinants. They all aimed to investigate theory of changing self-awareness theory and its aggression effect. Private and public self-awareness have influenced the behaviors of aggression in reference to the observed results and are validated by theory of Prentice-awareness. An individual seeking about himself and contemplating about his innate being and having sensations of awareness for his hidden dimensions is denoted by private self-awareness [6]. 
Application of several psychosocial interventions have been treated to resolve anger problems in the practice of mental health such as progressive muscle relaxation, biofeedback, meditation, relaxation, cognitive restructuring, self-instructional training, problem solving, social skills training, exposure, assertiveness training, stress inoculation and education. Numerous investigated studies have shown that these psychological mitigations are effective on anger reduction. For example, Snyder, Kymissis, and Kessler (1999) observed that psychosocial interventions are effective for anger and aggression. The outcomes showed that there was a remarkable reduction in the treatment group from pre- to post evaluation on the MMPI-Adolescent Anger Content Scale. In another investigation, Watt and Howells (1999) studied the psychosocial intervention effectiveness for violent offenders. Little support has been provided by the outcomes of participants for mitigation gains in intervening the condition relative to its regulation. Deffenbacher, Dahlen, et al. (2000) observed the effectiveness of psychosocial alleviation for reduction of anger in an undergraduate population. The outcomes showed a remarkable clinical change of several participants by meeting an intervention condition index [3].

Several meta-analyses have been facilitated for the efficacy determination of various intervention approaches where evidence by cognitive-behavioral approaches is observed to be the most effective means to mitigate anger-related issues. Metaanalytical investigation showed variations in the sizes of effect for several interventions within cognitive behavioral mitigations. Tafrate (1995) discovered the biggest size of effect for relaxation-based interventions, whereas DiGuiseppe and Tafrate (2003) showed the biggest size of effect for cognitive restructuring, following systematic desensitization. In a broad sense, the component for the most effective in the treatment of anger problems is unclear but certain conclusion states that cognitive and behavioral approaches have to be applied in training of anger management. On the grounds of meta-analytical review, Sukhodolsky and colleagues (2001), discovered moderate sizes of effect for various models of cognitive-behavioral intervention (CBI). In aggressive behavior reduction, social skills training and multimodal interventions were observed to be more effective, whereas, in subjective anger reduction due to experiences, problem solving approaches were found to be effective. Furthermore, the homework use was remarkably and positively associated to therapy results. Del Vecchio and O'Leary (2004) studied the anger management research that was facilitated in the past 2 decades and the superiority of cognitive treatments in mitigation of anger suppression whereas cognitive-behavioral intervention (CBI) in alleviation of anger expression issues [7]. 
Behaviors are recognized as defense principles during the analysis of a traumatized individual as defined by the symptoms associated to trauma. Early ideas regarding psychological trauma were concentrated physically on signs of psychological distress, while modern interventions are dealt on mental symptoms. However, traumatic experiences show evocation of psychobiological reactions. On the perspective of gradual development by Baldwin (2013), psycho-physiological responses to traumatic events have been developed to assure survival. Operation of primary mechanisms in survival conditions has been facilitated with intermittent cortical adjustments. The facilitation of these principles offers a ground for evaluating the co-morbidities and symptoms associated to trauma. Various early models suggest that the mammalian brain developed by the generation of novel neural circuits which is growing with complexity so as to permit the adjustment of the reflexes in paleocortex domains persuaded by the modern world [8]. This paper aimed to measure the severity of emotional injury as a result of the anger management done by the 33-year-old Asian man.

\section{Methodology}

Psychological intervention in trauma observed in post-traumatic anger management (PTAM) was the concentration of this research which is likely the most common outcome subsequent to a traumatic episode [8].

For a major mitigation analysis after traumatic episodes, a bibliographic search was performed. Investigations were only acknowledged if post-traumatic symptoms were the chief aim of psychological approach [8].

In order to make this investigation more particular, there was a consultation of other meta-analyses. The works from several consultations showed that few kinds of approaches were utilized more usual in comparison to others. According to this result, cognitive-behavioral intervention (CBI) and eye movement desensitization and reprocessing (EMDR) were utilized in several cases. Moreover, some therapists chose stress management as a method of mitigation [8].

In accordance with the Agency for Healthcare Research and Quality, a comparison has to be made between psychological and pharmacological approaches for prevention of post-traumatic symptoms in adults as shown in Figure 1.

Numerous particular kinds of psychological approaches to perform post-traumatic anger management (PTAM) in adults include psychological debriefing interventions including critical incident stress debriefing (CISD) and Critical Incident Stress Management (CISM), psychological first aid (PFA), cognitive restructuring mitigation, cognitive 


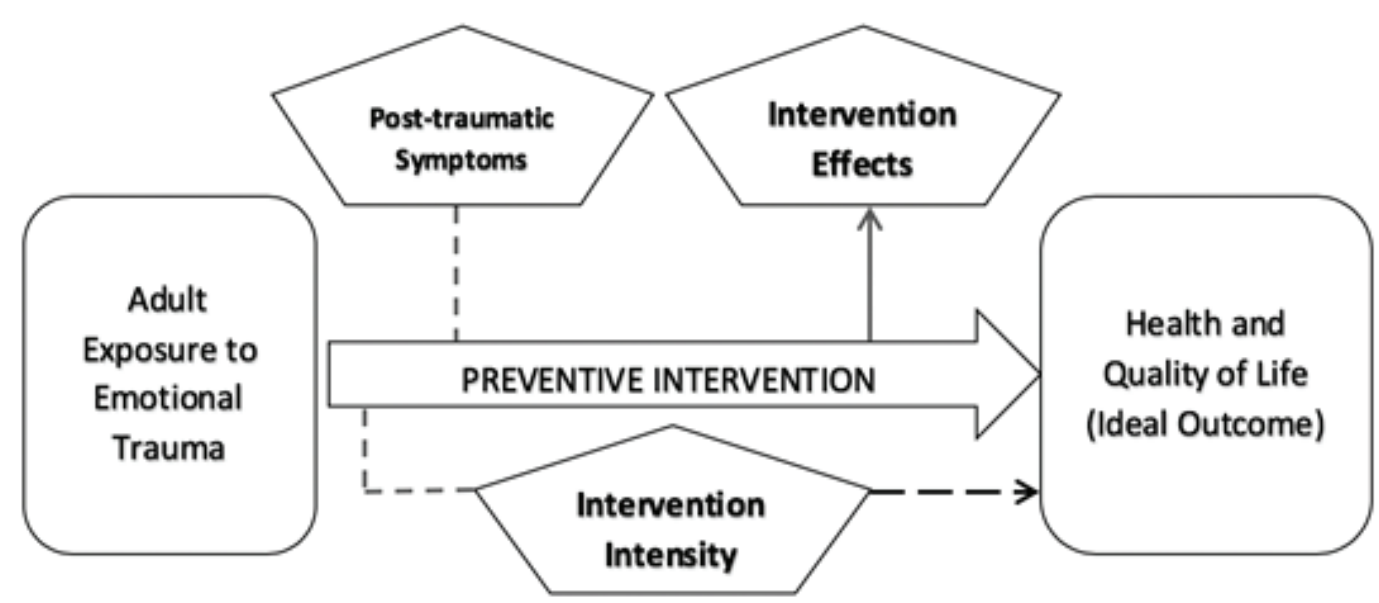

Figure 1: Analytical mechanism of intervention aiming for post-traumatic symptoms prevention in adults subsequent to psychological trauma exposure.

processing approach, exposure-based interventions, coping skills alleviation such as stress inoculation mitigation, psycho-education, eye movement desensitization and reprocessing (EMDR), cognitive-behavioral intervention (CBI). These interventions are designed for the prevention of trauma-related symptom development, shortly subsequent to a traumatic episode exposure [8].

Psychological debriefing approaches raise the victim awareness on normal reactions to trauma with encouragement to disseminate their experiences and emotional replies to the episode [8].

Commonly, conduction of facilitator-led group process for CISD is acknowledged for people under anger soon after a traumatic episode of 36-72 hours from exposure to emotional trauma. The process is commonly comprised of seven steps, namely, Introduction; Fact Phase; Thought Phase; Reaction Phase; Symptom Phase; Teaching Phase; and Re-entry Phase, when structured [8].

CISD is a secondary approach to prevention originally designed for utilization with people indirectly subjected to traumatic episodes, where a team comprised of people familiar with the association of mental health professionals performs its administration. In order to aid the victims to relieve their post-traumatic symptoms and to deal about their emotions, experience and behavior, the operators demonstrate coping skills and provides additional supplies. CISD intervention is flexible with a relaxed framework [8].

Although the purpose of CISD was not to promote post-traumatic anger management (PTAM), its application has been directly performed with the victims of trauma. Some investigations showed that this approach might be inefficient for that goal and actually may exhibit detrimental effects. Rose et al. (2002) revised and modified a past 
1997 Cochrane Review that evaluated the efficiency of single-session psychological debriefing for the intervention of psychological distress subsequent to trauma and posttraumatic symptom prevention [8].

CISD has been expanded and favorably changed into Critical Incident Stress Management (CISM), a multi-level and comprehensive crisis mitigation program with the aim of severity reduction for emotional trauma. CISM comprises more means and tools such as pre-incident training for individuals with high-risk works, one-on-one individual crisis assistance, demobilization such information about coping and stress to big clusters of emergency employees, and act of defusing such as small-group approached during which participants are inquired for exploration and discussion of their incident and emotional replies to it. CISM has a goal wherein family members of emergency workers have to be debriefed as well. Furthermore, referral processes for deploying individuals for psychological services should be executed [8].

Psychological first aid (PFA) comprises a cluster of aiding protocols to reduce first post-traumatic episode distress and assisting short and long-term adaptive variables. PFA is developed to serve as response to an initial step of a complex trauma and designed to perform eight essential actions, in reference to AHRQ 2012, namely, contact and engagement, safety and comfort, stabilization, information cluster, practical aid, association to social supports, information on coping support, and relation collaborative services. Major advantages can be observed by PFA implementation such as high portability and the likelihood of ubiquitous delivery where recent trauma survivors can be observed [8].

Cognitive-behavioral intervention (CBI) utilizes altering mechanisms of some ineffective beliefs and conditioning to intervene with post-traumatic symptoms and comprises of components coming from behavior and cognitive alleviation. Since CBI is utilized in several other stress disorders, for the traumatic episodes, there are particular approaches termed as trauma-focused $\mathrm{CBI}$, such as exposure, cognitive restructuring, and several coping skills, either alone or combined with the other. Majority of traumafocused CBI types are concise and include weekly sessions of around 60 to 90 minutes. This mechanism can be administered either as a cluster or individual intervention [8].

Exposure-based intervention exhibits confrontation with traumatic stimuli, process by process, and is continued until there is reduction in anxiety. The exposure is grounded on mental imagery from memory or administered in scenes demonstrated, in vitro, by the therapist. Some exposure are in vivo cases, especially when the victim has a weak imagination and the therapist discovers a very familiar context same with the traumatic one. The goal is for disappearance of the conditioned emotional response to traumatic 
stimuli by understanding that absence of negative notion will occur during traumatic episodes, which eventually lead to reduction or elimination of feared situation prevention and the affect related to it. Exposure intervention is usually facilitated for 8 to 12 weekly or biweekly appointments of around 60 to 90 minutes [8].

Cognitive restructuring is grounded on the ideal concept of personal perception of the episode, rather than the episode itself. This principle determines a person's mood, in reference to Epictetus and later, to Selye's method. Its aim is to operate and reposition the victim towards the distorted perceptions and beliefs spawned from a traumatic episode and augment the awareness of dysfunctional trauma-associated perceptions and correct or substitute those thoughts with more rational and/or adaptive cognitions. Cognitive restructuring is usually administered over 8 to 12 appointments of around 60 to 90 minutes [8].

Eye movement desensitization and reprocessing (EMDR) joins the imaginary exposure with accompanying influence of rhythmic eye movements, which are perceived to assist in reprogramming brain function, in order to reduce emotional impact of trauma and eventually, be worked out. In EMDR process, the client is advised to recall a traumatic memory and then, to solve on the incompatibility between positive and negative cognitions. The therapist suggests the client to meditate memory while concentrating on fast moving fingers by clinicians. Subsequent to 10 to 12 eye movements, the clinician suggests the client to evaluate the memory strength and related perceptions within the positive cognition frame. While earlier EMDR versions comprised of 1 to 3 appointments, existing standards show 8 to 12 appointments with 90 minutes every 7 days [8].

\section{Results and Discussion}

Age, sex, comorbidity, traumatism severity and hospital stay length have been correlated to quality and health of life. The traumatism severity is stratified in accordance to the Injury Severity Score index (ISS) which associated to mortality or negative event. An anatomical scoring system grounded on the Abbreviated Injury Scale (AIS) is termed as ISS that measures the injury severity in various anatomical domains [9].

The injury severity across the varying body domains can be described using the Abbreviated Injury Scale (AIS) system. Derived by agreement, personal injury by anatomic body domain can be categorized by global severity scoring system in accordance to its relative 6-point severity scale with a score of one (1) for minor, and six (6) for maximal. The Association for the Advancement of Automotive Medicine is the system responsible for its modification, expansion and enhancement. It has recently 
updated its latest modification, the AIS 2005 - Update 2008 and AIS 2015. The Injury Severity Score (ISS) was generated by Baker et al. in 1974 and has been recognized as the "gold standard" among severity indicators of anatomic injury and summarized the single patient's various injuries into a single score. Summation of the squares of the severity digit in the AIS of the most severe injuries, in three out of six or $50 \%$ predefined body domain based on AIS severity values [10].

In spite of its result to possible less severe injury inclusion in other body domains rather than additive serious injury in the same body region, the ISS solely constitutes one injury for each body domain. An altered ISS, the New Injury Severity Score (NISS), was acknowledged by Osler et al. in 1997 in order to answer its conflicting limitation. Moreover, Exponential Injury Severity Score (EISS) by Wang et al. in 2014 has generated an alteration of the AIS system. Calculation of the EISS is described as the basic variance of the AIS values by increment of each AIS severity score, to compute $1 \pm 6$, by 3 taking a power of AIS minus 2, and then, addition of the three (3) most severe scores or the highest AIS values, despite of body domains. Mathematical expression is exhibited as EISS $=3^{A-2}+3^{B-2}+3^{C-2}$, where A, B, and C are the three (3) most severe AIS codes [10].

A 33-year-old Asian obese individual who had suffered verbal abuse and oppression began to be aggressive in conversations and the intensity of his anger was managed by lifting heavy weights for several months. Existing and lost weights were measured every month and is tabulated below:

TABLE 1: Weight Summary of Existing and Lost Weights Per Month.

\begin{tabular}{l|l} 
MONTHLY WEIGHT: & KILOS LOST EVERY MONTH: \\
\hline September $2017-67$ kilos & Sept - Oct $2017-5$ kilos \\
\hline October $2017-62$ kilos & Oct - Nov $2017-4$ kilos \\
November $2017-58$ kilos & Nov - Dec $2017-1$ kilo \\
\hline December $2017-57$ kilos & Dec - Jan $2018-1$ kilo \\
\hline January $2018-56$ kilos & Jan - Feb $2018-1$ kilo \\
February $2018-55$ kilos & Feb - Mar $2018-2$ kilos \\
March $2018-53$ kilos & Mar - Apr $2018-3$ kilos \\
April $2018-50$ kilos & TOTAL -17 kilos
\end{tabular}

His anger kept on persisting as he recalls his previous encounters with past acquaintances. In order to control his anger, he kept on lifting heavy weights starting September of 2017 to April of 2018. Results of lost weight were shown in Table 1. In computation of his body mass index (BMI), the 33-year-old Asian man went from obese to normal BMI 
acknowledging a height of 58.27 inches, in a duration of 8 months. The EISS calculation of the 33-year-old Asian man is exhibited below:

Exponential Injury Severity Score Computation:

$$
\begin{aligned}
\text { EISS } & =3^{5-2}+3^{4-2}+3^{3-2} \\
& =3^{3}+3^{2}+3^{1} \\
& =27+9+3 \\
& =39
\end{aligned}
$$

EISS by Interpolation:

$$
\begin{gathered}
5=27 \\
X=39 \\
6=81 \\
\frac{x-5}{1}=\frac{12}{42} \\
x-5=\frac{12(1)}{42} \\
x=0.29+5
\end{gathered}
$$

$x=5.29($ SEVERE INJURY BASED ON TABLE 2)

TABLE 2: EISS Calculation according to the AIS Codes.

\begin{tabular}{|l|c|c|c|}
\hline AIS Codes (A) & $\mathbf{3}^{A-2}(\mathbf{B})$ & $(\mathbf{C})$ & (D) \\
\hline 1 & $3^{1-2}$ & $3^{-1}$ & 0.3 \\
\hline 2 & $3^{2-2}$ & $3^{0}$ & 1 \\
\hline 3 & $3^{3-2}$ & $3^{1}$ & 3 \\
\hline 4 & $3^{4-2}$ & $3^{2}$ & 9 \\
\hline 5 & $3^{5-2}$ & $3^{3}$ & 27 \\
\hline 6 & $3^{6-2}$ & $3^{4}$ & 81 \\
\hline
\end{tabular}

Cognitive-behavioral intervention (CBI) served as the approach chosen by the 33year-old Asian man to mitigate his post-traumatic symptoms of injustice leading to 
extreme anger exacerbated by abusive words resulting to emotional trauma. In order to alleviate his anger, vigorous lifting of weights was performed by the 33-year-old Asian man that resulted to significant weight loss exhibited as normal BMI from obese category, hence, leading back to an excellent and healthy life as shown in Figure 2.

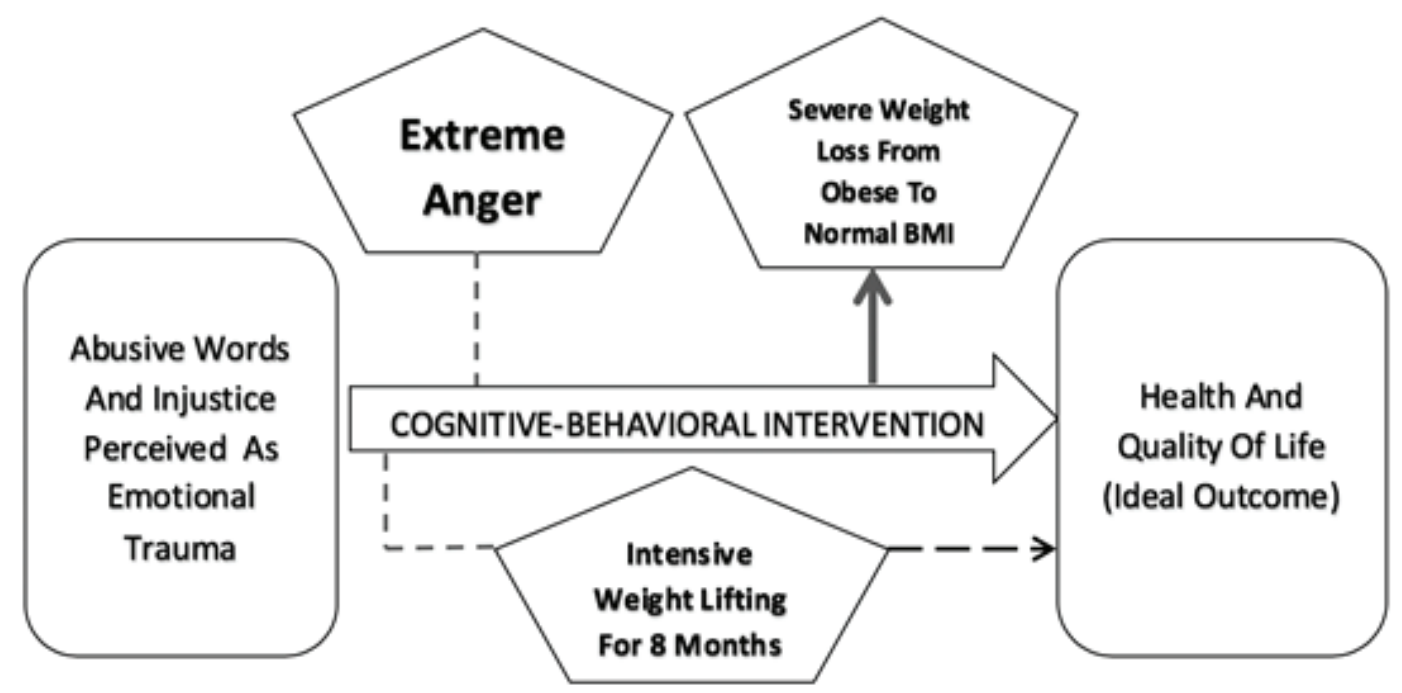

Figure 2: Analytical principle cognitive-behavioral intervention (CBI) aiming for the liberation of emotional trauma subsequent to exposure of emotional trauma.

\section{Conclusion}

Abbreviated Injury Scale (AIS) is the measurement of severity of injuries based on emotional trauma experienced by an individual. Exponential Injury Severity Score (EISS) is a modification tool of AIS by change computation in one body region pertaining to weight reduction. From 67 kilos to 50 kilos body weight loss observed from September 2017 to April 2018, computed EISS exhibited a score of 5.29 indicating a severe trauma experienced by the 33-year-old Asian man. Hence, management of anger can be executed through $\mathrm{CBI}$ in order to promote quality of health and life.

\section{References}

[1] Schneider, J. C., Trinh, N. T., Selleck, E., Fregni, F., Salles, S. S., Ryan, C. M., Stein, J. (2012). The Long-Term Impact of Physical and Emotional Trauma: The Station Nightclub Fire, Plos One, 7 (10):1-9.

[2] Thomas, S. C. (2002). Psychological Consequences of Traumatic Injury, British Journal of Psychiatry, 180, 392-393. 
[3] Valizadeh, S., Davaji, R. B. O., Nikamal, M. (2010). The effectiveness of anger management skills training on reduction of aggression on adolescents, Social and Behavioral Sciences, 5, 1195-1199.

[4] Shokoohi-Yekta, M., Zamani, N., Parand, A. (2010). Efficacy of anger management training based on cognitive-behavioral approach on mothers of children with mental retardation: 1-year follow-up, Social and Behavioral Sciences, 5, 1434-1437.

[5] Nasir, R. and Ghani, N. A. (2014). Behavioral and Emotional Effects of Anger Expression and Anger Management among Adolescents, Social and Behavioral Sciences, 140, 565-569.

[6] Mohammadiarya, A., Sarabi, S. D., Shirazi, M., Lachinani, F., Roustaei, A., Abbasi, Z., Ghasemzadeh, A. (2012). The effect of training self-awareness and anger management on aggression level in Iranian middle school students, Social and Behavioral Sciences, 46, 987-991.

[7] Akdas Mitrani, A. T. (2010). Outcome of anger management training program in a sample of undergraduate students, Social and Behavioral Sciences, 5, 339-344.

[8] Vasile, C (2014). An analysis of psychological trauma interventions, Social and Behavioral Sciences, 127, 781-785.

[9] Koo, M., Otero, I., Sabate, A., Martinez, R., Mauro, A., Garcia, P., Lopez, S. (2014). Do the severity and the body region of injury correlate with long-term outcome in the severe traumatic patient?, Revista Brasileira De Anestesiologia, 64 (2), 134-139.

[10] Kuo, S. C. H., Kuo, P. J., Chen, Y. C., Chien, P. C., Hsieh, H. Y., Hsieh, C. H. (2017). Comparison of the new Exponential Injury Severity Score with the Injury Severity Score and the New Injury Severity Score in trauma patients: A cross-sectional study, Plos One, 12 (11), 1-12.

Z. Llarena was born in Manila, Philippines. The author finished his BS Pharmacy degree at the University of Santo Tomas and graduated his MS Pharmacy Major in Pharmaceutical Chemistry at the University of the Philippines Manila. He is currently enrolled at De La Salle University to finish PhD in Chemical Engineering. He used to work both in the pharmaceutical industry and academe as Assistant Professor and Program Chair of the Pharmacy Department in colleges and universities in the Philippines. 